\title{
GLOBAL AND LOCAL OPTIMALITY CONDITIONS IN SET-VALUED OPTIMIZATION PROBLEMS
}

\author{
M. DUREA
}

Received 6 September 2004 and in revised form 18 April 2005

We consider both global and local conditions for optimization problems governed by setvalued maps. For global conditions, we present a comparative study and then we impose the weaker ones to obtain optimality conditions. Therefore, we introduce some local conditions and we prove that these conditions are useful (mainly) in the study of Borwein proper minima for the considered problems.

\section{Preliminaries}

Through the paper, $X$ and $Y$ are normed vector spaces; however, most of the results remain true in the more general setting of locally convex spaces. We denote by $X^{*}$ and $Y^{*}$ the topological dual spaces of $X$ and $Y$. We consider a pointed closed convex cone $Q \subset Y$ which introduces a partial order on $Y$ by the equivalence $y_{1} \leq_{Q} y_{2} \Leftrightarrow y_{2}-y_{1} \in Q$; we also suppose, in general, that $Q$ has nonempty interior. We set $Q^{+}:=\left\{y^{*} \in Y^{*} \mid y^{*}(y) \geq 0\right.$, $\forall y \in Q\}$ for the dual cone of $Q$ and $Q^{+i}:=\left\{y^{*} \in Y^{*} \mid y^{*}(y)>0, \forall y \in Q \backslash\{0\}\right\}$ for the quasi-interior of $Q^{+}$. We take a set-valued map $F$ from $X$ into $Y$. As usual, we denote the graph and domain of $F$, respectively, by

$$
\begin{gathered}
\operatorname{Gr} F=\{(x, y) \in X \times Y \mid y \in F(x)\}, \\
\operatorname{Dom} F=\{x \in X \mid F(x) \neq \varnothing\} .
\end{gathered}
$$

If $D$ is a subset of $X$, then $F(D):=\bigcup_{x \in D} F(x)$ and if $y \in Y, F^{-1}(y)=\{x \in X \mid y \in F(x)\}$.

We recall now the basic definitions used in the sequel.

Definition 1.1. Let $D$ be a nonempty subset of $X$ and $\bar{x} \in X$. The Bouligand tangent cone to $D$ at $\bar{x}$ (named the contingent cone in [2]) is the set

$$
T_{B}(D, \bar{x})=\left\{u \in X \mid \exists\left(t_{n}\right) \downarrow 0, \exists\left(u_{n}\right) \longrightarrow u, \forall n \in \mathbb{N}, \bar{x}+t_{n} u_{n} \in D\right\},
$$

where $\left(t_{n}\right) \downarrow 0$ means $\left(t_{n}\right) \subset(0, \infty)$ and $\left(t_{n}\right) \rightarrow 0$. 
The following properties are well known (see [2]):

(P1) $T_{B}(D, \bar{x})$ is a closed cone; if $D$ is convex, $T_{B}(D, \bar{x})$ is convex and $T_{B}(D, \bar{x})=\mathrm{C}(D-$ $\bar{x})$, where $\mathrm{C}(A)$ denotes the closed cone generated by a set $A$;

(P2) $T_{B}(D, \bar{x})=T(\operatorname{cl} D, \bar{x})$ and $T_{B}(D, \bar{x})=T_{B}(D \cap V, \bar{x})$ for every neighborhood $V$ of $\bar{x}$.

Definition 1.2. Let $(\bar{x}, \bar{y}) \in \mathrm{Gr} F$. The Bouligand derivative of $F$ at $(\bar{x}, \bar{y})$ is the set-valued map $D_{B} F(\bar{x}, \bar{y})$ from $X$ into $Y$ defined by

$$
\operatorname{Gr}\left(D_{B} F(\bar{x}, \bar{y})\right)=T_{B}(\operatorname{Gr} F,(\bar{x}, \bar{y})) .
$$

The following definition was introduced by Penot [26] and is also used in [28].

Definition 1.3. Let $(\bar{x}, \bar{y}) \in \mathrm{Gr} F$. The Dini lower derivative of $F$ at $(\bar{x}, \bar{y})$ in direction $u$ is given by

$$
\begin{gathered}
\underline{D F}(\bar{x}, \bar{y})(u)=\left\{v \in Y \mid \forall\left(t_{n}\right) \downarrow 0, \forall\left(u_{n}\right) \longrightarrow u, \exists\left(v_{n}\right) \longrightarrow v, \exists n_{0} \in \mathbb{N},\right. \\
\left.\forall n \geq n_{0}, \bar{y}+t_{n} v_{n} \in F\left(\bar{x}+t_{n} u_{n}\right)\right\} .
\end{gathered}
$$

Let $M \subset Y$ be a nonempty set; we consider the following minimum concepts.

Definition 1.4. (i) (see [24]) An element $y \in M$ is called $Q$-minimum for $M$ if $(M-y) \cap$ $(-Q)=\{0\}$.

(ii) (see [24]) An element $\in M$ is called $Q$-weak minimum for $M$ if $(M-y) \cap(-\operatorname{int} Q)$ $=\varnothing$.

(iii) (see [24]) An element $y \in M$ is called $Q$-proper minimum for $M$ if there exists a convex cone $P$ such that $Q \backslash\{0\} \subset \operatorname{int} P$ and $y$ is $P$-minimum for $M$.

(iv) (see [4]) An element $y \in M$ is called $Q$-proper efficient in the sense of Borwein for $M$ if $T_{B}(M+Q, y) \cap(-Q)=\{0\}$ (see also [19]).

(v) (see [3]) An element $y \in M$ is called Q-proper efficient in the sense of Benson for $M$ if $\mathrm{C}(M+Q-y) \cap(-Q)=\{0\}$.

We denote the set of $Q$-minimum points for $M$ by $\operatorname{Min}(M, Q)$. The sets of other minimum elements considered in the above definition are, respectively, $W \operatorname{Min}(M, Q)$, $P \operatorname{Min}(M, Q), \operatorname{Bo} \operatorname{Min}(M, Q)$, and $\operatorname{Be} \operatorname{Min}(M, Q)$. The next inclusions are obvious:

$$
P \operatorname{Min}(M, Q) \subset \operatorname{Min}(M, Q) \subset W \operatorname{Min}(M, Q) .
$$

From property (P1), we have

$$
\operatorname{Be} \operatorname{Min}(M, Q) \subset \operatorname{Bo} \operatorname{Min}(M, Q) \subset \operatorname{Min}(M, Q) .
$$

In the sequel, we use some cone separation theorems. We recall that a cone $S$ is said to have a base $B$ if $B$ is convex, $0 \notin \mathrm{cl} B$, and $S=\mathbb{R}_{+} B$.

Theorem 1.5 (see [8]). Let $P$ and $S$ be cones in $Y, P \cap S=\{0\}$. If $P$ is closed and $S$ has a compact base, then there exists a pointed convex cone $K$ such that $S \backslash\{0\} \subset \operatorname{int} K$ and $P \cap K=$ $\{0\}$. 
Theorem 1.6 (see [4]). Let $P$ and $S$ be convex, closed cones in $Y$ with $P \cap S=\{0\}$ and $S$ have a compact base. Then $\left(-P^{+}\right) \cap S^{+i} \neq \varnothing$.

The following auxiliary result is inspired by [6, Lemma 2.5].

Lemma 1.7. Let $M \subset Y$ be a nonempty set, $y \in M$, and let $V$ be an open, convex neighborhood of $y$. Then the next equalities hold:

$$
\begin{aligned}
& \operatorname{cl}((M+Q) \cap V)=\operatorname{cl}((M+\operatorname{int} Q) \cap V), \\
& \operatorname{int}(\operatorname{cl}((M+Q) \cap V))=(M+\operatorname{int} Q) \cap V .
\end{aligned}
$$

Proof. In (1.7) the inclusion " $\supset$ " is obvious. Let $z \in(M+Q) \cap V, v \in \operatorname{int} Q$, and let $W$ be an open neighborhood of $z$. Then $V \cap W$ is also a neighborhood of $z$; moreover, there exists $a \in M$ such that $z-a \in Q$. Consequently, there exists $\alpha \in(0,1)$, close enough to 1 , such that $\alpha z+(1-\alpha)(v+a) \in V \cap W$, that is, $a+(1-\alpha) v+\alpha(z-a) \in V \cap W$. Since $(1-\alpha) v+\alpha(z-a) \in \operatorname{int} Q$, we have that $(M+\operatorname{int} Q) \cap V \cap W \neq \varnothing$. The neighborhood $W$ was arbitrarily chosen, hence $z \in \operatorname{cl}((M+\operatorname{int} Q) \cap V)$ and the first equality follows.

Let us prove the second relation. Since $(M+\operatorname{int} Q) \cap V \subset \operatorname{cl}((M+Q) \cap V)$ and the first set is open, the inclusion " $\supset$ " is clear. Conversely, we take $z \in \operatorname{int}(\operatorname{cl}((M+Q) \cap V))$ and $v \in$ int $Q$; there exists a symmetric neighborhood $W$ of 0 such that $z-W \subset \mathrm{cl}((M+$ $Q) \cap V)$. But $W$ is absorbing, hence there exists $\alpha>0$ with $\alpha v \in W$. The set $z-\operatorname{int} Q$ is a neighborhood of $z-\alpha v$, hence $(z-\operatorname{int} Q) \cap((M+Q) \cap V) \neq \varnothing$. This implies that $z \in M+\operatorname{int} Q$. Of course, $z \in V$ because $z \in \operatorname{int}(\operatorname{cl}((M+Q) \cap V)) \subset \operatorname{int} \operatorname{cl} V=V$. This proves (1.8).

We present below a characterization of weak minima in terms of contingent cone (see $[9,10])$. For the sake of completeness, we prove this assertion.

Proposition 1.8. A point $y \in W \operatorname{Min}(M, Q)$ if and only if

$$
T_{B}(M+Q, y) \cap(-\operatorname{int} Q)=\varnothing .
$$

Proof. Suppose by contradiction that $y \in W \operatorname{Min}(M, Q)$ and there exists $v \in T_{B}(M+$ $Q, y) \cap(-\operatorname{int} Q)$; then there exist $\left(t_{n}\right) \downarrow 0$ and $\left(v_{n}\right) \rightarrow v$ such that for $n$ large enough, $y+t_{n} v_{n} \in M+Q$. But, for a large $n, v_{n} \in-\operatorname{int} Q$, hence $t_{n} v_{n} \in-$ int $Q$. Consequently, $y \in M+Q+\operatorname{int} Q \subset M+\operatorname{int} Q$, a contradiction.

For the converse, suppose that (1.9) holds and that there exists $a \in M$ such that $a-y \in$ - int $Q$ and take $\left(t_{n}\right) \downarrow 0$. For $n$ large enough, $t_{n}<1$, so

$$
a+\left(1-t_{n}\right)(y-a) \in M+\operatorname{int} Q
$$

that is,

$$
y+t_{n}(a-y) \in M+\operatorname{int} Q,
$$

which shows that

$$
a-y \in T_{B}(M+\operatorname{int} Q, \bar{y}) .
$$


But, taking $V=Y$ in relation (1.8), we have $\mathrm{cl}(M+\operatorname{int} Q)=\operatorname{cl}(M+Q)$. On the other hand, from (P2), we can write

$$
a-y \in T_{B}(\operatorname{cl}(M+\operatorname{int} Q), y)=T_{B}(\operatorname{cl}(M+Q), y)=T_{B}(M+Q, y) .
$$

Since $a-y \in-\operatorname{int} Q$, this is in contradiction with (1.9).

\section{Subconvexity for set-valued maps}

The study of multiobjective optimization problems involving set-valued maps (as well as those involving vector-valued functions) has developed in the recent years some generalizations of convexity concepts. Among these generalizations, a widely used class of concepts is the so-called convexlikeness which contains in the case of set-valued maps at least four items used in the papers $[11,14,18,20,21,22]$. The main purpose of this section is to complete this class and to study the connections between these concepts; also we prove here an alternative Gordan-Farkas-type theorem which generalizes some results in [22] and this gives us the possibility to extend in a more general setting some necessary optimality conditions for weak minimum points from $[14,21,22]$.

The first result of this section is an easy consequence of Lemma 1.7.

Lemma 2.1. Let $M$ be a nonempty subset of $Y$. Then $M+\operatorname{int} Q$ is convex if and only if $\mathrm{cl}(M+Q)$ is convex.

Proof. Suppose that $M+\operatorname{int} Q$ is convex; then $\operatorname{cl}(M+\operatorname{int} Q)$ is convex as well and, following relation (1.8) with $V=Y$, the $\operatorname{set} \operatorname{cl}(M+Q)$ is convex. If $\operatorname{cl}(M+Q)$ is convex, then $\operatorname{int}(\mathrm{cl}(M+Q))$ is convex and, following relation (1.7) with $V=Y$, we have the conclusion.

Let $A$ be a nonempty subset of $X$, included in Dom $F$. We present the first of the generalized convexity concepts which we study in the sequel and which is used, for example, in $[21,22]$.

Definition 2.2. The set-valued map $F$ is called $Q$ subconvex on $A$ if there exists $\varphi \in \operatorname{int} Q$, such that for all $\varepsilon>0, \alpha \in(0,1)$, and $x_{1}, x_{2} \in A$, there exists $x_{3} \in A$ with $\varepsilon \varphi+\alpha F\left(x_{1}\right)+$ $(1-\alpha) F\left(x_{2}\right) \subset F\left(x_{3}\right)+Q$.

The next generalization of the convexity for set-valued map is also used in [22]. For the single-valued case, this notion is used, for example, in [23].

Definition 2.3. The set-valued map $F$ is called $Q$ pseudoconvex on $A$ if for all $\alpha \in(0,1)$, and $x_{1}, x_{2} \in A$, there exists $x_{3} \in A$ such that $\alpha F\left(x_{1}\right)+(1-\alpha) F\left(x_{2}\right) \subset F\left(x_{3}\right)+Q$.

The next notion is introduced in [1].

Definition 2.4. A set $C \subset X$ is called nearly convex if there exists $\alpha \in(0,1)$ such that $\alpha x_{1}+(1-\alpha) x_{2} \in C$ for all $x_{1}, x_{2} \in C$.

In [1] it is proved that if $C$ is nearly convex, then the set $\left\{\beta \in[0,1] \mid \forall x_{1}, x_{2} \in C\right.$, $\left.\beta x_{1}+(1-\beta) x_{2} \in C\right\}$ is dense in $[0,1]$. Using a simple sequence-based argument, it results that $\mathrm{cl} C$ is convex. In [6] the notion of nearly convex function is defined. A similar 
concept for set-valued maps is used in [27]. We introduce now some concepts based on Definition 2.4.

Definition 2.5. The set-valued map $F$ is called

(a) $Q$ nearly subconvex on $A \subset X$ if there exists $\alpha \in(0,1)$, and $\varphi \in \operatorname{int} Q$, such that for all $\varepsilon>0, x_{1}, x_{2} \in A$, there exists $x_{3} \in A$ with $\varepsilon \varphi+\alpha F\left(x_{1}\right)+(1-\alpha) F\left(x_{2}\right) \subset F\left(x_{3}\right)+$ Q;

(b) $Q$ nearly pseudoconvex on $A \subset X$ if there exists $\alpha \in(0,1)$, such that for all $x_{1}, x_{2} \in$ A, there exists $x_{3} \in A$ with $\alpha F\left(x_{1}\right)+(1-\alpha) F\left(x_{2}\right) \subset F\left(x_{3}\right)+Q$.

In order to establish the relations between the above concepts and some convexity assumptions widely used in the literature, we start with a characterization result.

Proposition 2.6. for all $\psi \in \operatorname{int} Q, \alpha \in(0,1), x_{1}, x_{2} \in A$, and $y_{1} \in F\left(x_{1}\right), y_{2} \in F\left(x_{2}\right)$, there exists $x_{3} \in A$ such that $\psi+\alpha y_{1}+(1-\alpha) y_{2} \in F\left(x_{3}\right)+\operatorname{int} Q$.

Proof. The equivalence between (i) and (ii) follows from Lemma 2.1.

We prove now that (i) implies (iii). Let $\psi \in \operatorname{int} Q, \alpha \in(0,1), x_{1}, x_{2} \in A$, and $y_{1} \in F\left(x_{1}\right)$, $y_{2} \in F\left(x_{2}\right)$. Therefore,

$$
\begin{aligned}
\psi+\alpha y_{1}+(1-\alpha) y_{2} & =\alpha\left(y_{1}+\psi\right)+(1-\alpha)\left(y_{2}+\psi\right) \\
& \in \alpha(F(A)+\operatorname{int} Q)+(1-\alpha)(F(A)+\operatorname{int} Q) \\
& \subset F(A)+\operatorname{int} Q,
\end{aligned}
$$

the conclusion.

We prove that (iii) implies (i). Let $v_{1}, v_{2} \in F(A)+$ int $Q$ and $\alpha \in(0,1)$; then there exist $x_{1}, x_{2} \in A, y_{1} \in F\left(x_{1}\right), y_{2} \in F\left(x_{2}\right)$, and $q_{1}, q_{2} \in \operatorname{int} Q$ such that $v_{1}=y_{1}+q_{1}$ and $v_{2}=y_{2}+$ $q_{2}$. Therefore,

$$
\alpha v_{1}+(1-\alpha) v_{2}=\alpha y_{1}+(1-\alpha) y_{2}+\alpha q_{1}+(1-\alpha) q_{2}
$$

Since int $Q$ is convex, $\alpha q_{1}+(1-\alpha) q_{2} \in \operatorname{int} Q$, hence there exists a neighborhood $V$ of the origin in $Y$ such that $\alpha q_{1}+(1-\alpha) q_{2}+V \subset \operatorname{int} Q$. From (iii), for every $\psi \in \operatorname{int} Q$, there exists $x_{3} \in A$ with $\psi+\alpha y_{1}+(1-\alpha) y_{2} \in F\left(x_{3}\right)+Q$; taking $\psi$ such that $-\psi \in V$ (there exists such an element because $V$ is absorbing and int $Q$ is closed with respect to the scalar multiplication), we have

$$
\alpha v_{1}+(1-\alpha) v_{2} \in F\left(x_{3}\right)+Q+\alpha q_{1}+(1-\alpha) q_{2}-\psi
$$

But $\alpha q_{1}+(1-\alpha) q_{2}-\psi \in \operatorname{int} Q$ and, from $Q+\operatorname{int} Q \subset \operatorname{int} Q$, we get

$$
\alpha v_{1}+(1-\alpha) v_{2} \in F\left(x_{3}\right)+\operatorname{int} Q \subset F(A)+\operatorname{int} Q
$$

that is, the conclusion. 
We give now a characterization of the $Q$ subconvexity.

Proposition 2.7. The next assertions are equivalent:

(i) $F$ is $Q$ subconvex on $A$;

(ii) for all $\psi \in \operatorname{int} Q$, for all $\alpha \in(0,1)$, for all $x_{1}, x_{2} \in A$, there exists $x_{3} \in A$ such that $\psi+\alpha F\left(x_{1}\right)+(1-\alpha) F\left(x_{2}\right) \in F\left(x_{3}\right)+\operatorname{int} Q$.

Proof. First we prove that (i) implies (ii). Let $\psi \in \operatorname{int} Q, \alpha \in(0,1), x_{1}, x_{2} \in A$. There exists a neighborhood $V$ of the origin in $Y$ such that $\psi+V \subset \operatorname{int} Q$. For the element $\varphi \in \operatorname{int} Q$ from the definition of $Q$ subconvexity, we take $\varepsilon>0$ such that $-\varepsilon \varphi \in V$. Therefore,

$$
\psi+\alpha F\left(x_{1}\right)+(1-\alpha) F\left(x_{2}\right) \in F\left(x_{3}\right)+Q+\psi-\varepsilon \varphi \subset F\left(x_{3}\right)+\operatorname{int} Q
$$

The converse is obvious.

Remark 2.8. A consequence of the above results is that if $F$ is $Q$ subconvex on $A$, then, necessarily, $F(A)+$ int $Q$ is convex. Similarly, one can prove the following assertions: if $F$ is $Q$ pseudoconvex on $A$, then $F(A)+Q$ is convex, and if $F$ is $Q$ nearly pseudoconvex on $A$, then $F(A)+Q$ is nearly convex. If $F$ is $Q$ nearly subconvex on $A$, then the set $F(A)+\operatorname{int} Q$ is nearly convex; but $F(A)+\operatorname{int} Q$ is an open set and, taking into account the properties of nearly convex sets, this is equivalent with the convexity of $F(A)+\operatorname{int} Q$. Consequently, all generalizations of the convexity defined above imply the convexity of $F(A)+\operatorname{int} Q$.

In the sequel, our aim is to give examples which show that the reverse implications in the above remark are not always true. We present three main examples. The first example shows that a set-valued map for which $F(A)+Q$ is convex is not necessarily $Q$ pseudoconvex on $A$.

Example 2.9. Let $X=\mathbb{R}, Y=\mathbb{R}^{2}, Q=\mathbb{R}_{+}^{2}:=\left\{(y, z) \in \mathbb{R}^{2} \mid y \geq 0, z \geq 0\right\}, A=\{1,2\}$. We take $F(1)=\left\{(y, z) \in \mathbb{R}^{2} \mid y+z \geq 1, y \in[0,1 / 2], z \in[1 / 2,1]\right\}$ and $F(2)=\left\{(y, z) \in \mathbb{R}^{2} \mid\right.$ $y+z \geq 1, y \in[1 / 2,1], z \in[0,1 / 2]\}$. Then

$$
\begin{aligned}
& F(1)+\mathbb{R}_{+}^{2}=\left\{(y, z) \in \mathbb{R}^{2} \mid y+z \geq 1, y \geq 0, z \geq \frac{1}{2}\right\}, \\
& F(2)+\mathbb{R}_{+}^{2}=\left\{(y, z) \in \mathbb{R}^{2} \mid y+z \geq 1, y \geq \frac{1}{2}, z \geq 0\right\}, \\
& F(A)+\mathbb{R}_{+}^{2}=\left\{(y, z) \in \mathbb{R}^{2} \mid y+z \geq 1, y \geq 0, z \geq 0\right\} .
\end{aligned}
$$

It is clear that $F(A)+\mathbb{R}_{+}^{2}$ is convex. Let $\alpha=1 / 2$ and the points $(0,1),(1 / 2,1 / 2) \in F(1)$, $(1 / 2,1 / 2),(1,0) \in F(2)$. Therefore, $\alpha(0,1)+(1-\alpha)(1 / 2,1 / 2)=(1 / 4,3 / 4) \in F(1)+\mathbb{R}_{+}^{2}$, but $(1 / 4,3 / 4) \notin F(2)+\mathbb{R}_{+}^{2}$. On the other hand, $\alpha(1 / 2,1 / 2)+(1-\alpha)(1,0)=(3 / 4,1 / 4) \in F(2)+$ $\mathbb{R}_{+}^{2}$, but $(3 / 4,1 / 4) \notin F(1)+\mathbb{R}_{+}^{2}$, which means that $F$ is not $Q$ pseudoconvex on $A$.

Moreover, we can prove that $F$ is not $Q$ nearly pseudoconvex on $A$. Suppose that there exists $\alpha \in(0,1)$ such that $\alpha F(1)+(1-\alpha) F(2) \subset F(1)+\mathbb{R}_{+}^{2}$, or $\alpha F(1)+(1-\alpha) F(2) \subset$ $F(2)+\mathbb{R}_{+}^{2}$. Let $\left(y_{1}, z_{1}\right) \in F(1)$, and $\left(y_{2}, z_{2}\right) \in F(2)$; then $\alpha y_{1}+(1-\alpha) y_{2}$ can take any value in $[1 / 2-\alpha / 2,1-\alpha / 2]$, and $\alpha z_{1}+(1-\alpha) z_{2}$ can take any value in $[\alpha / 2,1 / 2+\alpha / 2]$, 
and $\alpha y_{1}+(1-\alpha) y_{2}+\alpha z_{1}+(1-\alpha) z_{2} \geq 1$. Since $[1 / 2-\alpha / 2,1-\alpha / 2] \nsubseteq[1 / 2, \infty)$, it implies that $\alpha F(1)+(1-\alpha) F(2) \nsubseteq F(2)+\mathbb{R}_{+}^{2}$; in the same way, $[\alpha / 2,1 / 2+\alpha / 2] \nsubseteq[1 / 2, \infty)$, hence $\alpha F(1)+(1-\alpha) F(2) \nsubseteq F(1)+\mathbb{R}_{+}^{2}$. This shows that $F$ is not $Q$ nearly pseudoconvex on $A$. Of course, being convex, $F(A)+Q$ is nearly convex.

Since $F(A)+Q$ is nearly convex, $F(A)+\operatorname{int} Q$ is convex. We show that $F$ is not $Q$ nearly subconvex on $A$. Suppose the contrary and take $\left(\varphi_{1}, \varphi_{2}\right) \in \operatorname{int} Q\left(\varphi_{1}, \varphi_{2}>0\right)$ and $\alpha \in(0,1)$, the elements from Definition 2.5(a). Let $\varepsilon>0$ such that $\varepsilon \varphi_{1} \in(0, \alpha / 2)$ and $\varepsilon \varphi_{2} \in(0,(1-$ $\alpha) / 2)$. Using the same argument as above, we have that $\varepsilon \varphi+\alpha F(1)+(1-\alpha) F(2) \nsubseteq F(1)+$ $\mathbb{R}_{+}^{2}$ and $\varepsilon \varphi+\alpha F(1)+(1-\alpha) F(2) \nsubseteq F(2)+\mathbb{R}_{+}^{2}$, a contradiction.

Although we can give examples to show that there exist set-valued maps which are $Q$ nearly pseudoconvex on a given set and are not $Q$ pseudoconvex on that set, one can see that this is proved in the case of single-valued maps in [6, Remark 4.3] and it is sufficient to prove the assertions also for set-valued maps.

Example 2.10. Let $X=\mathbb{R}, Y=\mathbb{R}^{2}, Q=\mathbb{R}_{+}^{2}:=\left\{(y, z) \in \mathbb{R}^{2} \mid y \geq 0, z \geq 0\right\}, A=[0,1]$. We consider

$$
F(x)= \begin{cases}\{(y, z) \mid y+z \geq 1, y=x\}, & \text { if } x \in A \backslash\left\{\frac{1}{2}\right\}, \\ \{(y, z) \mid y+z>1, y=x\}, & \text { if } x=\frac{1}{2} .\end{cases}
$$

We have

$$
F(A)+Q=\{(y, z) \mid y+z \geq 1, y \geq 0, z \geq 0\} \backslash\left\{\left(\frac{1}{2}, \frac{1}{2}\right)\right\}
$$

which is not convex. But

$$
F(A)+\operatorname{int} Q=\{(y, z) \mid y+z>1, y>0, z>0\}
$$

is convex. On the other hand, $F$ is not $Q$ pseudoconvex on $A$, but it is $Q$ subconvex on $A$ because for every $\psi \in \operatorname{int} Q, \alpha \in(0,1)$, and $x_{1}, x_{2} \in A$, we have $\psi+\alpha F\left(x_{1}\right)+(1-$ $\alpha) F\left(x_{2}\right) \subset F\left(\alpha x_{1}+(1-\alpha) x_{2}\right)+\operatorname{int} Q$ and $\alpha x_{1}+(1-\alpha) x_{2} \in A$.

Example 2.11. Let $X=\mathbb{R}, Y=\mathbb{R}^{2}, Q=\left\{(y, z) \in \mathbb{R}^{2} \mid y \geq 0, z \geq 0, y \leq z\right\}, A=\mathbb{R}$. We consider

$$
F(x)= \begin{cases}\{x\} \times \mathbb{R}_{+}, & \text {if } x \in \mathbb{R} \backslash \mathbb{Q} \\ \{x\} \times \mathbb{R}_{+}^{*}, & \text { if } x \in \mathbb{Q} .\end{cases}
$$

Then

$$
F(A)+Q=\{(y, z) \mid y>0\} \cup\{(x, 0) ; x \in \mathbb{R} \backslash \mathbb{Q}\},
$$


which is not nearly convex because $\mathbb{R} \backslash \mathbb{Q}$ is not nearly convex (so $F$ is not nearly pseudoconvex), and

$$
F(A)+\operatorname{int} Q=\{(y, z) \mid y>0\}
$$

is convex. Moreover, it is easy to observe that $F$ is $Q$ nearly subconvex on $A$.

We consider now an optimization problem with set-valued maps which was studied for the first time in the seminal paper of Corley [7]:

(P)

$$
\min F(x) \text { subject to } x \in A, G(x) \cap(-R) \neq \varnothing,
$$

where $F$ and $G$ are set-valued maps, $G: X \rightrightarrows Z$ ( $Z$ is a normed vector space), and $R \subset Z$ is a pointed convex cone with nonempty interior.

Definition 2.12. An element $(\bar{x}, \bar{y}) \in \mathrm{Gr} F$ is called weak solution of problem (2.13) if $\bar{x} \in$ $A, G(x) \cap(-R) \neq \varnothing$ and $\bar{y}$ is a weak minimizer of $F(A)$, that is, $(F(A)-\bar{y}) \cap(-\operatorname{int} Q)=$ $\varnothing$.

We present two theorems which, together, give us a Gordan-Farkas alternative result. Taking into account the above discussion, we impose general convexity assumptions.

Theorem 2.13. Suppose that

(i) $(F \times G)(A)+\operatorname{int}(Q \times R)$ is convex and for every $x \in A,(F \times G)(x) \cap(-\operatorname{int} Q \times$ $-\operatorname{int} R)=\varnothing$, or

(ii) $(F \times G)(A)+\operatorname{int} Q \times R$ is nearly convex and for every $x \in A,(F \times G)(x) \cap(-\operatorname{int} Q \times$ $-R)=\varnothing$.

Then there exists $\left(y^{*}, z^{*}\right) \in Q^{+} \times R^{+},\left(y^{*}, z^{*}\right) \neq(0,0)$, such that for every $x \in A, y \in$ $F(x), z \in G(x)$, it holds that $y^{*}(y)+z^{*}(z) \geq 0$.

If, moreover, the Slater condition $G(A) \cap(-\operatorname{int} R) \neq \varnothing$ holds, then $y^{*} \neq 0$.

Proof. In case (i), we have $(0,0) \notin(F \times G)(A)+\operatorname{int} Q \times \operatorname{int} R$ and this set is convex. In case (ii), $(0,0) \notin(F \times G)(X)+\operatorname{int} Q \times R$ and this set is nearly convex, so $\operatorname{int}((F \times G)(A)+$ int $Q \times R)$ is convex and $(0,0) \notin \operatorname{int}((F \times G)(A)+\operatorname{int} Q \times R)$; from Lemma 1.7, we obtain $(0,0) \notin(F \times G)(A)+\operatorname{int} Q \times$ int $R$. In both cases, we can apply the Eidelheit separation theorem: there exists $\left(y^{*}, z^{*}\right) \in Y^{*} \times Z^{*},\left(y^{*}, z^{*}\right) \neq(0,0)$, such that for all $x \in A, y \in$ $F(x), z \in G(x), \varphi \in \operatorname{int} Q, \chi \in \operatorname{int} R$,

$$
y^{*}(y)+z^{*}(z)+y^{*}(\varphi)+z^{*}(\chi) \geq 0
$$

If there exists $\varphi \in$ int $Q$ with $y^{*}(\varphi)<0$, taking the element $n \varphi \in \operatorname{int} Q$ for $n \in \mathbb{N}$ large enough in relation (2.14), we arrive at a contradiction. Consequently, $y^{*}(\varphi) \geq 0$ for every $\varphi \in \operatorname{int} Q$, so $y^{*}(\varphi) \geq 0$ for every $\varphi \in \operatorname{cl}(\operatorname{int} Q)=\operatorname{cl} Q \supset Q$, that is, $y^{*} \in Q^{+}$. Similarly, $z^{*} \in R^{+}$. Taking $\varphi \rightarrow 0$ and $\chi \rightarrow 0$ in relation (2.14), we have $y^{*}(y)+z^{*}(z) \geq 0$. If the Slater condition holds, and $y^{*}=0$, then $z^{*}(z) \geq 0$ for every $z \in G(A)$, hence, in particular, also for $z \in G(A) \cap(-\operatorname{int} R)$, a contradiction. The proof is complete. 
Theorem 2.14. If there exists $\left(y^{*}, z^{*}\right) \in Q^{+} \times R^{+},\left(y^{*}, z^{*}\right) \neq(0,0)$, such that for every $x \in A, y \in F(x), z \in G(x)$, it holds that $y^{*}(y)+z^{*}(z) \geq 0$, then for all $x \in A,(F \times G)(x) \cap$ $(-\operatorname{int} Q \times-\operatorname{int} R)=\varnothing$.

If there exists $\left(y^{*}, z^{*}\right) \in Q^{+} \times R^{+}, y^{*} \neq 0$, such that for every $x \in A, y \in F(x), z \in G(x)$, it holds that $y^{*}(y)+z^{*}(z) \geq 0$, then for all $x \in A,(F \times G)(x) \cap(-\operatorname{int} Q \times-R)=\varnothing$.

Proof. We prove only the second part, the first being similar. We suppose by contradiction that there exists $x \in A$ such that $(F \times G)(x) \cap(-$ int $Q \times-R) \neq \varnothing$. Then there exist $y \in F(x) \cap(-\operatorname{int} Q)$ and $z \in G(x) \cap(-R)$ with $y^{*}(y)+z^{*}(z) \geq 0$. But $y^{*} \neq 0$ and $y \in$ - int $Q$ imply that $y^{*}(y)<0$; on the other hand, $z^{*}(z) \leq 0$, which is a contradiction.

These two results cover [14, Theorem 3.1], [21, Theorem 2.1], and [22, Proposition $3]$. As usual, an alternative result leads to an optimality result. We give bellow our version which extends the corresponding results from the quoted papers.

Theorem 2.15. Suppose that

(i) $(F \times G)(A)+\operatorname{int}(Q \times R)$ is convex, or

(ii) $(F \times G)(A)+$ int $Q \times R$ is nearly convex.

If $(\bar{x}, \bar{y})$ is a weak solution of problem (2.13), then there exists $\left(y^{*}, z^{*}\right) \in Q^{+} \times R^{+},\left(y^{*}, z^{*}\right)$ $\neq(0,0)$, such that for every $x \in A, y \in F(x), z \in G(x)$, it holds that $y^{*}(y)+z^{*}(z) \geq y^{*}(\bar{y})$ and $z^{*}(v)=0$ for all $v \in G(\bar{x}) \cap(-R)$. If, moreover, the Slater condition holds, then $y^{*} \neq 0$.

Proof. It is clear that if $F$ and $G$ satisfy assumption (i) or (ii), then the set-valued map $F-\bar{y}$ given by $(F-\bar{y})(x):=F(x)-\bar{y}$ and $G$ satisfy the same assumption. Since $(\bar{x}, \bar{y})$ is a weak solution of problem (2.13), there is no $x \in A$ such that $((F-\bar{y}) \times G) \cap(-\operatorname{int} Q \times$ $-R) \neq \varnothing$. We can apply Theorem 2.13: there exists $\left(y^{*}, z^{*}\right) \in Q^{+} \times R^{+},\left(y^{*}, z^{*}\right) \neq(0,0)$, such that for every $x \in A, y \in F(x), z \in G(x)$, we have

$$
y^{*}(y-\bar{y})+z^{*}(z) \geq 0
$$

and the first part of the conclusion follows. Let $v \in G(\bar{x}) \cap(-R)$; since $z^{*} \in R^{+}, z^{*}(v) \leq 0$. On the other hand, taking $y=\bar{y}$ in (2.15), we obtain $z^{*}(v) \geq 0$. Of course, the Slater condition ensures that $y^{*} \neq 0$.

We consider now problem (2.13) with $A=X$, denoted by $(\dot{\mathrm{P}})$. We have the following result.

Theorem 2.16. Let $(\bar{x}, \bar{y})$ be a weak solution of problem $(\dot{P})$ and $\bar{z} \in G(\bar{x}) \cap(-R)$. If the set $\left.D_{B} F(\bar{x}, \bar{y}) \times \underline{D} G(\bar{x}, \bar{z})\right)(X)+\operatorname{int}(Q \times R)$ is convex, then there exists $\left(y^{*}, z^{*}\right) \in Q^{+} \times R^{+}$, $\left(y^{*}, z^{*}\right) \neq(0,0)$, such that for every $u \in X, v \in D_{B} F(\bar{x}, \bar{y})(u), z \in \underline{D} G(\bar{x}, \bar{z})(u)$, it holds that $y^{*}(v)+z^{*}(z) \geq 0$.

Proof. Suppose by contradiction that there exists $u \in X$ such that

$$
D_{B} F(\bar{x}, \bar{y})(u) \times \underline{D} G(\bar{x}, \bar{z})(u) \cap(-\operatorname{int} Q \times-\operatorname{int} R) \neq \varnothing,
$$


that is, there exist $v \in D_{B} F(\bar{x}, \bar{y})(u)$ and $z \in \underline{D} G(\bar{x}, \bar{z})(u)$ such that $v \in-\operatorname{int} Q$ and $z \in$ $-\operatorname{int} R$. Following the definitions, there exist $t_{n} \downarrow 0, u_{n} \rightarrow u, v_{n} \rightarrow v$ such that $\bar{y}+t_{n} v_{n} \in$ $F\left(\bar{x}+t_{n} u_{n}\right)$ and $z_{n} \rightarrow z$ such that $\bar{z}+t_{n} z_{n} \in G\left(\bar{x}+t_{n} u_{n}\right)$. But

$$
\bar{z}+t_{n} z_{n} \in-R-\operatorname{int} R \subset-\operatorname{int} R,
$$

for $n$ large enough. Therefore, $G\left(\bar{x}+t_{n} u_{n}\right) \cap(-\operatorname{int} R) \neq \varnothing$. Since $(\bar{x}, \bar{y})$ is a weak solution of problem $\left(P^{\prime}\right)$, we deduce that

$$
\left(F\left(\bar{x}+t_{n} u_{n}\right)-\bar{y}\right) \cap(-\operatorname{int} Q)=\varnothing .
$$

Consequently, $t_{n} v_{n} \notin-$ int $Q$ for every $n$ large enough, a contradiction. We apply Theorem 2.13 and we obtain the conclusion.

A similar result holds if we impose conditions as in Theorem 2.13(ii) for $D_{B} F(\bar{x}, \bar{y})$ and $\underline{D} G(\bar{x}, \bar{z})$.

\section{Local optimality conditions}

In the recent years, many authors have studied optimization problems involving setvalued maps using generalized convexity concepts with good stability properties which are helpful in order to write optimality conditions for the studied problems (see $[5,12$, $13,15,16,17,23,24,25,27])$. In the majority of the quoted papers, the generalizations of the convexity for sets, and for set-valued maps as well, have a global character in the sense that depends on the entire shape of the admissible set or of the image through the set-valued map of the feasible points set. The basic idea of this section is to exploit the fact that there are minimum notions and tangent cones notions which depend only on the shape of admissible points in a neighborhood of the reference point. In this way, we present some new local concepts which can be possible candidates to replace global conditions like those studied in the preceding section, in the study of minimum notions involving tangent cones.

We present some basic definitions and results that are required in the sequel and we introduce the notions of strictly star-shaped set and nearly convex set at a point. Under conditions based on these concepts, we obtain some linear scalarization results concerning Borwein's proper minimum and the equivalence of this type of minima with other types of proper minimum. We use the following additional notations: if $y^{*} \in Y^{*}$, we write $\left\langle A, y^{*}\right\rangle$ instead of $\left\{\left\langle a, y^{*}\right\rangle \mid a \in A\right\}$ and $\left\langle A, y^{*}\right\rangle \geq 0$ if $\left\langle a, y^{*}\right\rangle \geq 0$ for all $a \in A$; similarly, we write $\left\langle A, y^{*}\right\rangle \geq\left\langle y, y^{*}\right\rangle$ if $\left\langle a, y^{*}\right\rangle \geq\left\langle y, y^{*}\right\rangle$ for all $a \in A$ and for some $y \in Y$. The first local concept is defined below.

Definition 3.1. Let $M \subset Y$ be a nonempty set and $y \in M$.

(i) $M$ is called strictly star shaped at $y$ if there exists $q \in(0,1)$ such that for all $a \in M$, there exists $\lambda \in(0, q]$ with $(1-\lambda) y+\lambda a \in M$.

(ii) Let $p, q \in(0,1), p \leq q$. $M$ is called $(p, q)$-star shaped at $y$ if for all $a \in M$, there exists $\lambda \in[p, q]$ such that $(1-\lambda) y+\lambda a \in M$. 
Of course, a $(p, q)$-star-shaped set at a point is strictly shaped at that point and a set is $(p, p)$-star shaped at every point if and only if it is $p$-convex. So the notion of star-shaped set at a point is rather general.

We have the following proposition on the above concepts.

Proposition 3.2. (i) If $M$ is $(p, q)$-star shaped at $y$, then $\operatorname{cl} M$ is $(p, q)$-star shaped at $y$.

(ii) $M$ is strictly star shaped at $y$ if and only if for every convex set $B$ and for all $b \in B$, the set $M+B$ is strictly star shaped at $y+b$.

Proof. (i) Take $\bar{a} \in \operatorname{cl} M$; there exists a sequence $\left(a_{n}\right) \subset M$ such that $a_{n} \rightarrow \bar{a}$. From definition, one can find $\left(\lambda_{n}\right) \subset[p, q]$ such that

$$
\left(1-\lambda_{n}\right) y+\lambda_{n} a_{n} \in M
$$

Since $\left(\lambda_{n}\right)$ is a bounded sequence in $\mathbb{R}$, we can suppose (without relabeling) that it is convergent to some $\lambda \in[p, q]$. Passing to the limit in relation (3.1), we obtain that (1ג) $y+\lambda \bar{a} \in \operatorname{cl} M$.

(ii) Suppose that $M$ is strictly star shaped at $y$ and take $B$ a convex set, $b \in B$ and $z=c+d$ with $c \in M, d \in B$; from Definition 3.1, there exist $q \in(0,1)$ and $\lambda \in(0, q]$ such that $(1-\lambda) y+\lambda c \in M$. Since $B$ is convex, $(1-\lambda) b+\lambda d \in B$, and adding these relations, we have that $(1-\lambda)(y+b)+\lambda z \in M+B$, that is, $M+B$ is strictly star shaped at $y+b$. For the converse implication, take $B=\{0\}$.

The main property of strictly star-shaped sets which we use in the sequel is given in the next result.

Proposition 3.3. If $M$ is strictly star shaped at $y$, then $T_{B}(M, y)=\mathrm{C}(M-y)$.

Proof. Taking into account properties (P1) and (P2), we only have to show that $M$ is pseudoconvex at $y$ (see [2, page 145]), that is, $M-y \subset T_{B}(M, y)$. Take $a \in M$; the set

$$
M_{(y, a)}=\{\lambda \in(0, q] \mid(1-\lambda) y+\lambda a \in M\}
$$

contains at least an element $\lambda^{a}$. Hence, $a_{1}=\left(1-\lambda^{a}\right) y+\lambda^{a} a \in M$; from definition, there exists $\lambda^{a_{1}} \in(0, q]$ such that

$$
\left(1-\lambda^{a_{1}}\right) y+\lambda^{a_{1}} a_{1} \in M
$$

that is,

$$
\left(1-\lambda^{a_{1}}\right) y+\lambda^{a_{1}}\left(\left(1-\lambda^{a}\right) y+\lambda^{a} a\right) \in M
$$

hence

$$
\left(1-\lambda^{a} \lambda^{a_{1}}\right) y+\lambda^{a} \lambda^{a_{1}} a \in M .
$$

It is clear that $0<\lambda^{a} \lambda^{a_{1}} \leq q^{2}$ and $\lambda^{a} \lambda^{a_{1}} \neq \lambda^{a}$; from relation (3.5), $\lambda^{a} \lambda^{a_{1}} \in M_{(y, a)}$. In a similar way, we can construct now a sequence $\left(\lambda_{n}\right) \subset M_{(y, a)}$ such that $0<\lambda_{n} \leq q^{n}$ for all $n$, hence $\left(\lambda_{n}\right) \downarrow 0$. But $\left(1-\lambda_{n}\right) y+\lambda_{n} a \in M$ means that $y+\lambda_{n}(a-y) \in M$, that is, $a-y \in$ $T_{B}(M, y)$. The element $a$ was arbitrarily chosen in $M$, so $M-y \subset T_{B}(M, y)$ and this is the conclusion. 
Practically, in the proof of the above proposition, we proved that $M$ is strictly starshaped at $y$ if and only if

$$
\forall a \in M, \quad \exists\left(\lambda_{n}\right) \subset(0,1), \lambda_{n} \rightarrow 0 \quad \text { s.t. } \forall n,\left(1-\lambda_{n}\right) y+\lambda_{n} a \in M .
$$

As a first consequence of Proposition 3.3, we obtain the following corollary.

Corollary 3.4. If the set $M+Q$ is strictly star shaped at $y \in M$, then $y \in \operatorname{BoMin}(M, Q)$ if and only if $y \in \operatorname{Be} \operatorname{Min}(M, Q)$.

We mention that in the literature the coincidence of these minimum concepts is given only in global convexity assumptions. In our corollary, the involved cones are not necessarily convex.

Following Proposition 3.2(ii), the assumption that $M+Q$ is strictly star shaped at $y$ is weaker than the assumption that $M$ is strictly star shaped at $y$. For example, consider $Q=[0, \infty) \times\{0\} \subset \mathbb{R}^{2}$ and $M \subset \mathbb{R}^{2}, M=\{0\} \times[0,2] \cup\{(x, y) \mid x \geq 0, y \geq 0, x+y \leq 1\} \cup$ $\{(x, y) \mid x \geq 0, y \in[1,2], x-y \leq-1\}$. The set $M$ is not strictly star shaped at $(1,0)$, but $M+Q=[0, \infty) \times[0,2]$ is convex.

We prove that under appropriate assumptions PMin and Bo Min are the same.

Proposition 3.5. (i) $P \operatorname{Min}(M, Q) \subset \operatorname{BoMin}(M, Q)$;

(ii) if $M+Q$ is strictly star shaped at $y \in M$, $Q$ has compact base, and $y \in \operatorname{BoMin}(M, Q)$, then $y \in P \operatorname{Min}(M, Q)$.

Proof. (i) Let $y \in P \operatorname{Min}(M, Q)$; then there exists a convex cone $P$ such that $Q \backslash\{0\} \subset \operatorname{int} P$ and $y \in \operatorname{Min}(M, P) \subset W \operatorname{Min}(M, P)$. Following Proposition 1.8, $T_{B}(M+P, y) \cap(-\operatorname{int} P)$ $=\varnothing$, which implies that $T_{B}(M+Q, y) \cap(-Q)=\{0\}$. Hence, $y \in \operatorname{BoMin}(M, Q)$.

(ii) Let $y \in \operatorname{Bo} \operatorname{Min}(M, Q)$, that is, $T_{B}(M+Q, y) \cap(-Q)=\{0\}$. We can apply Theorem 1.5, so there exists a convex, pointed cone $P$ such that $T_{B}(M+Q, y) \cap(-P)=$ $\{0\}$ and $Q \backslash\{0\} \subset \operatorname{int} P$. Again, in our assumptions, $M-y \subset M+Q-y \subset T_{B}(M+Q, y)$, hence $(M-y) \cap(-P)=\{0\}$, that is, $y \in P \operatorname{Min}(M, Q)$.

Using Corollary 3.4 and Proposition 3.5, we obtain the next result.

COROLlARY 3.6. If $M+Q$ is strictly star shaped at $y \in M$ and $Q$ has compact base, then the following are equivalent:

(i) $y \in \operatorname{BoMin}(M, Q)$;

(ii) $y \in \operatorname{Be} \operatorname{Min}(M, Q)$;

(iii) $y \in P \operatorname{Min}(M, Q)$.

We present now another notion which we will use in the sequel.

Definition 3.7. $M$ is called nearly convex at $y$ if there exists an open convex neighborhood $V$ of $y$ such that $\operatorname{cl}(M \cap V)$ is convex.

Remark 3.8. If $M$ is nearly convex at $y$, then $T_{B}(M, y)$ is convex, because, following (P2), one has $T_{B}(M, y)=T_{B}(M \cap V, y)=T_{B}(\operatorname{cl}(M \cap V), y)$ and the last set is convex from (P1). 
We present now a scalarization result for Borwein proper minimum.

Proposition 3.9. Suppose that $M+Q$ is nearly convex at $y \in M$ and $Q$ has compact base. If $y \in \operatorname{Bo} \operatorname{Min}(M, Q)$, then there exists $y^{*} \in Q^{+i}$ such that

$$
\left\langle T_{B}(M+Q, y), y^{*}\right\rangle \geq 0 .
$$

In particular, $\left\langle M \cap V, y^{*}\right\rangle \geq\left\langle y, y^{*}\right\rangle$, where $V$ is the neighborhood of $y$ from Definition 3.7. If, moreover, $M+Q$ is strictly star shaped at $y$, then $\left\langle M, y^{*}\right\rangle \geq\left\langle y, y^{*}\right\rangle$. Conversely, if there exists $y^{*} \in Q^{+i}$ such that $\left\langle M, y^{*}\right\rangle \geq\left\langle y, y^{*}\right\rangle$, then $y \in \operatorname{BoMin}(M, Q)$.

Proof. The first part results from Theorem 1.6, because in our assumptions $T_{B}(M+Q, y)$ is a convex cone (see Remark 3.8). In particular, $M \cap V-y \subset(M+Q) \cap V-y \subset T_{B}(M+$ $Q, y)$ and then $\left\langle v, y^{*}\right\rangle \geq\left\langle y, y^{*}\right\rangle$ for all $v \in M \cap V$.

If $M+Q$ is strictly star shaped at $y, T_{B}(M+Q, y)=\mathrm{C}(M+Q-y)$ and this proves that $\left\langle v, y^{*}\right\rangle \geq\left\langle y, y^{*}\right\rangle$ for all $v \in M$. The converse is true as well: if $\left\langle v-y, y^{*}\right\rangle \geq 0$ for all $v \in M$, then $\left\langle v-y, y^{*}\right\rangle \geq 0$ for all $v \in \mathrm{C}(M+Q-y)$, hence $T_{B}(M+Q, y) \cap(-Q)=\{0\}$ and this is the definition of $y \in \operatorname{Bo} \operatorname{Min}(M, Q)$.

In the sequel, we use mainly the condition that $M+Q$ is nearly locally convex at $y \in M$. We have the following result.

Lemma 3.10. Suppose that the cone $Q$ has nonempty interior. Then the set $M+Q$ is nearly convex at $y \in M$ if and only if there exists an open convex neighborhood $V$ of $y$ such that $(M+\operatorname{int} Q) \cap V$ is convex.

Proof. Suppose that $M+Q$ is nearly convex at $y$; there exists an open, convex neighborhood $V$ of $y$ such that $\operatorname{cl}((M+Q) \cap V))$ is convex, hence its nonempty interior is convex as well; relation (1.8) shows that $(M+\operatorname{int} Q) \cap V$ is convex. Suppose that $(M+\operatorname{int} Q) \cap V$ is convex; then its closure is convex as well, and relation (1.7) shows that $M+Q$ is nearly convex at $y$. The proof is complete.

\section{Application: Borwein proper minima}

In this section, as an application of the results presented in the previous section, we study the proper minimizers in the sense of Borwein for optimization problem (2.13). Let $X$ and $Z$ be normed vector spaces and $R$ a convex pointed cone in $Z$ with nonempty interior. In the first part of this section, we consider the following minimization problem:

(П)

$$
\min F(x) \text { subject to } x \in A \text {, }
$$

where $F: X \rightrightarrows Y$ is a set-valued map and $A \subset X$ is a nonempty set. A point $(x, y) \in X \times Y$ is called minimizer for problem (4.1) if $x \in A, y \in F(x)$, and $y \in \operatorname{Min}(F(A), Q)$. We work with similar definitions for the other minimum notions defined in Section 1. In order to apply some results of Section 3, we present a condition which ensures that $F(A)+Q$ is nearly convex at a point. 
Definition 4.1. $F$ is said to be subconvexlike at $y \in F(A)$ with respect to $A$ if there exists an open convex neighborhood $V$ of $y$ such that

$$
\begin{gathered}
\forall \alpha \in(0,1), \forall y_{1}, y_{2} \in F(A) \cap(V-Q), \\
\exists \varphi \in \operatorname{int} Q \text { s.t. } \forall \varepsilon>0, \varepsilon \varphi+\alpha y_{1}+(1-\alpha) y_{2} \in F(A)+\operatorname{int} Q .
\end{gathered}
$$

Proposition 4.2. Suppose that $y \in F(A)$. Consider the following affirmations:

(i) $F$ is subconvexlike at $y$ with respect to $A$;

(ii) there exists an open convex neighborhood $V$ of $y$ such that

$$
\forall \alpha \in(0,1), \forall y_{1}, y_{2} \in F(A) \cap(V-Q), \quad \alpha y_{1}+(1-\alpha) y_{2} \in \mathrm{cl}(F(A)+Q) ;
$$

(iii) $F(A)+Q$ is nearly convex at $y$.

Then (i) $\Leftrightarrow$ (ii) $\Rightarrow$ (iii).

Proof. (i) $\Rightarrow$ (ii). Take $V$ the same neighborhood as in Definition 4.1 and $\alpha \in(0,1), y_{1}, y_{2}$ $\in F(A) \cap(V-Q)$. Consider $\left(\varepsilon_{n}\right) \downarrow 0$; then there exists $\varphi \in \operatorname{int} Q$ such that $\varepsilon_{n} \varphi+\alpha y_{1}+$ $(1-\alpha) y_{2} \in F(A)+\operatorname{int} Q$ for all $n$. As $\varepsilon_{n} \varphi \rightarrow 0$, we can write

$$
\alpha y_{1}+(1-\alpha) y_{2} \in \operatorname{cl}(F(A)+\operatorname{int} Q)=\operatorname{cl}(F(A)+Q) .
$$

The last equality follows from (1.7) taking $V=Y$ and $M=F(A)$. See also [6, Lemma 2.5].

(ii) $\Rightarrow(\mathrm{i})$. Take $\alpha \in(0,1), y_{1}, y_{2} \in F(A) \cap(V-Q)$, and $\psi \in$ int $Q$. In our hypothesis, $\alpha y_{1}+(1-\alpha) y_{2} \in \operatorname{cl}(F(A)+Q)$, so

$$
\psi+\alpha y_{1}+(1-\alpha) y_{2} \in \mathrm{cl}(F(A)+Q)+\operatorname{int} Q .
$$

It is enough to prove that

$$
\mathrm{cl}(F(A)+Q)+\operatorname{int} Q=F(A)+\operatorname{int} Q
$$

to obtain an apparently stronger conclusion, because for all $\varphi \in \operatorname{int} Q$ and $\varepsilon>0, \varepsilon \varphi \in \operatorname{int} Q$. Indeed, the inclusion $F(A)+\operatorname{int} Q \subset \operatorname{cl}(F(A)+Q)+\operatorname{int} Q$ is obvious. Take $y \in \operatorname{cl}(F(A)+$ $Q)+\operatorname{int} Q$; then there exist $q \in \operatorname{int} Q,\left(y_{n}\right) \subset F(A)$, and $\left(q_{n}\right) \subset Q$ such that $y_{n}+q_{n} \rightarrow y-q$, that is, $y_{n}+q_{n}+q \rightarrow y$. As $\left(q_{n}+q\right) \subset$ int $Q$, we obtain that $y \in \operatorname{cl}(F(A)+Q)$. Then

$$
\mathrm{cl}(F(A)+Q)+\operatorname{int} Q \subset \mathrm{cl}(F(A)+\operatorname{int} Q) \subset \mathrm{cl}(F(A)+Q) .
$$

But $\operatorname{cl}(F(A)+Q)+\operatorname{int} Q$ is an open set, hence

$$
\mathrm{cl}(F(A)+Q)+\operatorname{int} Q \subset \operatorname{int}(\operatorname{cl}(F(A)+Q))=F(A)+\operatorname{int} Q .
$$

For the last equality, we used relation (1.8) for $V=Y$ and $F(A)$ instead of $M$.

(i) $\Rightarrow$ (iii). Taking into account Lemma 3.10, it is enough to prove that $(F(A)+\operatorname{int} Q) \cap$ $V$ is convex, where $V$ is the neighborhood of $y$ from Definition 4.1. Consider $v_{1}, v_{2} \in$ $(F(A)+\operatorname{int} Q) \cap V$ and $\alpha \in(0,1)$; there exist $y_{1}, y_{2} \in F(A), u_{1}, u_{2} \in \operatorname{int} Q$ such that $v_{i}=$ $y_{i}+u_{i}, i \in\{1,2\}$, hence $y_{i}=v_{i}-u_{i} \in V-\operatorname{int} Q=V-Q$. From Definition 4.1, there exists $\varphi \in \operatorname{int} Q$ such that for every $\varepsilon>0, \varepsilon \varphi+\alpha y_{1}+(1-\alpha) y_{2} \in F(A)+\operatorname{int} Q$, hence

$$
\varepsilon \varphi+\alpha v_{1}+(1-\alpha) v_{2}-\alpha u_{1}-(1-\alpha) u_{2} \in F(A)+\operatorname{int} Q
$$


We can find an $\varepsilon>0$ such that $\varepsilon \varphi-\alpha u_{1}-(1-\alpha) u_{2} \in-\operatorname{int} Q$, hence

$$
\alpha v_{1}+(1-\alpha) v_{2} \in F(A)+\operatorname{int} Q+\operatorname{int} Q=F(A)+\operatorname{int} Q
$$

and the proof is complete.

In fact, we proved that $F$ is subconvexlike at $y$ with respect to $A$ if and only if there exists an open convex neighborhood $V$ of $y$ such that

$$
\begin{gathered}
\forall \alpha \in(0,1), \forall y_{1}, y_{2} \in F(A) \cap(V-Q), \forall \psi \in \operatorname{int} Q, \\
\psi+\alpha y_{1}+(1-\alpha) y_{2} \in F(A)+\operatorname{int} Q .
\end{gathered}
$$

The implication from (iii) to (i) in the above result is false as the following example shows.

Take $F: \mathbb{R} \rightrightarrows \mathbb{R}^{2}, A=[0,4], Q=\mathbb{R}_{+}^{2}$, and

$$
F(x)= \begin{cases}\{x\} \times[0,1], & \text { if } x \in[0,1), \\ \{x\} \times[-1,1], & \text { if } x \in[1,2], \\ \varnothing, & \text { if } x \in(2,3), \\ \{x\} \times[3-x, x-3], & \text { if } x \in[3,4] .\end{cases}
$$

It is easy to see that $F$ is nearly convex at $(3,0)$ but, for every neighborhood $V$ of $(3,0)$, we can find $y_{1}, y_{2} \in F(A) \cap(V-Q)$, close to $(1,0)$, and $\alpha \in(0,1), \psi \in \operatorname{int} Q$, such that $\psi+\alpha y_{1}+(1-\alpha) y_{2} \notin F(A)+\operatorname{int} Q$.

Remark 4.3. If we take $V=Y$, it is easy to see that the condition of subconvexlikeness at a point is equivalent with the definition of subconvexlikeness (see, e.g., [22]) and is also equivalent with the convexity of $F(A)+$ int $Q$. So the subconvexlikeness at a point can be seen as a localization of the concept of subconvelikeness.

From Propositions 3.9 and 4.2, we obtain the next corollary.

Corollary 4.4. Suppose that $F$ is subconvexlike at $y \in F(A)$ with respect to $A$ and $Q$ has compact base. If $(x, y)$ is a proper minimizer in the sense of Borwein for problem (4.1), then there exists $y^{*} \in Q^{+i}$, such that $\left\langle T_{B}(F(A)+Q, y), y^{*}\right\rangle \geq 0$. If, moreover, $F(A)+Q$ is strictly star shaped at $y$, then $(x, y)$ is proper minimizer in the sense of Borwein for problem (4.1) if and only if

$$
\left\langle F(A), y^{*}\right\rangle \geq\left\langle y, y^{*}\right\rangle,
$$

for some $y^{*} \in Q^{+i}$.

Remark 4.5. In the assumptions we consider in the above corollary, $Q$ has compact base and has nonempty interior, the normed vector space $Y$ is necessarily finite dimensional. To prove this, we denote by $B$ the compact base of $Q$. Then the set $[0,1] B:=\{\alpha b \mid \alpha \in$ $[0,1], b \in B\}$ is a compact set with nonempty interior, so we can find a closed ball of $Y$ which is compact, hence $Y$ is finite dimensional. 
We illustrate the preceding corollary by some examples in which we consider nonconvex cases for problems of type (4.1). These examples show that the imposed conditions cannot be omitted and, on the other hand, have the role to emphasize the enlargement provided by our result to the usual case of such problems.

Example 4.6. Consider $F: \mathbb{R} \rightrightarrows \mathbb{R}^{2}, A=\mathbb{R}, Q=\left\{(x, u) \in \mathbb{R}^{2} \mid 0 \leq x \leq u / 2\right\}$. First we define

$$
F(x)= \begin{cases}\varnothing, & \text { if } x<-1 \text { or } x>0, \\ \{x\} \times([-1, x] \cup[-x, 1]), & \text { if } x \in[0,1] .\end{cases}
$$

Then $T_{B}(F(A)+Q,(0,0))=F(A)+Q$ and $(F(A)+Q) \cap-Q=\{0\}$, whence $(0,(0,0))$ is a proper minimizer in the sense of Borwein. But $F(A)+Q$ is not nearly convex at $y=(0,0)$ and $F(A)+Q$ cannot be included in the positive hyperplane given by some $y^{*} \in Q^{+i}$, so the subconvexlikeness condition cannot be omitted. Secondly, we take

$$
F(x)= \begin{cases}(x, 2), & \text { if } x<0, \\ \{x\} \times([0, x] \cup[2-x, 2]), & \text { if } x \in[0,1] \cap \mathbb{Q}, \\ \{x\} \times([0, x) \cup[2-x, 2]), & \text { if } x \in[0,1] \cap \mathbb{R} \backslash \mathbb{Q}, \\ \varnothing, & \text { if } x>1 .\end{cases}
$$

It is clear that $F$ is subconvexlike at $y=(0,0)$ with respect to $A$. Moreover, some calculations show that $T_{B}(F(A)+Q, y)=\left\{(x, y) \in \mathbb{R}^{2} \mid 0 \leq x \leq u\right\}$; therefore, $(0,(0,0))$ is a proper minimizer in the sense of Borwein. Consequently, we can use only the first part of our preceding corollary to include $T_{B}(F(A)+Q, y)$ in a hyperplane given by some $y^{*} \in Q^{+i}$, despite the nonconvexity of $F(A)+Q$. It is easy to see that $F(A)+Q$ is not strictly star shaped at $(0,0)$ and that $F(A)$ and $\{y\}$ cannot be separated by a hyperplane given by a $y^{*} \in Q^{+i}$, so in the second part of the above result, the strict star-shaped condition cannot be omitted. Now, if we take $Q=\mathbb{R}^{2}$ and

$$
F(x)= \begin{cases}\varnothing, & \text { if } x<0 \text { or } x>1, \\ \{x\} \times([0, x] \cup[2-x, 2]), & \text { if } x \in[0,1] \cap \mathbb{Q}, \\ \{x\} \times([0, x) \cup[2-x, 2]), & \text { if } x \in[0,1] \cap \mathbb{R} \backslash \mathbb{Q},\end{cases}
$$

we can apply the second part of the corollary as well in order to characterize Borwein minima.

Let us consider problem (2.13) from Section 2, where $G: X \rightrightarrows Z$ is a set-valued map. The definitions for minimizers of problem (2.13) are the same as for problem (4.1) with the set $K:=A \cap\{x \in X \mid G(x) \cap(-R) \neq \varnothing\}$ instead of $A$.

In order to prove a necessity result for problem (2.13), we need a condition to ensure the convexity of the set

$$
L:=\bigcup_{x \in A}((F(x)+\operatorname{int} Q) \cap V) \times(G(x)+\operatorname{int} R),
$$

where the notations are the same as above and $V$ is an open convex neighborhood of $y$. 
As in the proof of Proposition 4.2, it can be proved that the following assumption, which in a sense is a local subconvexlikeness condition for the pair $(F, G)$, ensures the desired convexity condition:

$$
\begin{gathered}
\forall \alpha \in(0,1), \forall x_{1}, x_{2} \in A, \forall y_{i} \in F\left(x_{i}\right) \cap(V-\operatorname{int} Q), \\
\forall z_{i} \in G\left(x_{i}\right), i \in\{1,2\}, \exists x_{3} \in A, \exists \varphi \in \operatorname{int} Q, \exists \psi \in \operatorname{int} R \text { s.t. } \forall \varepsilon>0, \\
\varepsilon \varphi+\alpha y_{1}+(1-\alpha) y_{2} \in F\left(x_{3}\right)+\operatorname{int} Q, \\
\varepsilon \psi+\alpha z_{1}+(1-\alpha) z_{2} \in G\left(x_{3}\right)+\operatorname{int} R .
\end{gathered}
$$

For related conditions used in the literature, see, for example, [13].

Theorem 4.7. Let $(x, y)$ be a proper minimizer in the sense of Borwein for the problem (2.13). Suppose that $F$ is subconvexlike at $y \in F(K)$ with respect to $K$ and $L$ is convex. Then there exist $\left(y^{*}, z^{*}\right) \in Q^{+} \times R^{+} \backslash\{(0,0)\}$ and an open convex neighborhood $V$ of $y$ such that, for all $x^{\prime} \in A$,

$$
\left\langle F\left(x^{\prime}\right) \cap V, y^{*}\right\rangle+\left\langle G\left(x^{\prime}\right), z^{*}\right\rangle \geq\left\langle y, y^{*}\right\rangle
$$

and $0 \in\left\langle G(x), z^{*}\right\rangle$.

Proof. First, we can consider that the neighborhoods of $y$ involved in subconvexlikeness and in the definition of the set $L$ are the same: simply take the intersection in both conditions and denote it by $V$. We have $T_{B}(F(K)+Q, y) \cap(-Q)=\{0\}$, hence $\mathrm{C}((F(K)+Q) \cap$ $V-y) \cap(-Q)=\{0\}$. Consequently,

$$
y \notin((F(K)+Q) \cap V)+\operatorname{int} Q .
$$

We claim that $(y, 0) \notin \bigcup_{x^{\prime} \in A}\left(\left(F\left(x^{\prime}\right)+\operatorname{int} Q\right) \cap V+\operatorname{int} Q\right) \times\left(G\left(x^{\prime}\right)+\operatorname{int} R\right)$. Indeed, in the contrary case, there exists $x^{\prime} \in A$ such that

$$
y \in\left(F\left(x^{\prime}\right)+\operatorname{int} Q\right) \cap V+\operatorname{int} Q, \quad 0 \in G\left(x^{\prime}\right)+\operatorname{int} R .
$$

The second inclusion ensures that $x^{\prime} \in K$ and the first that

$$
y \in(F(K)+\operatorname{int} Q) \cap V+\operatorname{int} Q,
$$

in contradiction with (4.20). But, from (A), the set

$$
\bigcup_{x^{\prime} \in A}\left(\left(F\left(x^{\prime}\right)+\operatorname{int} Q\right) \cap V+\operatorname{int} Q\right) \times\left(G\left(x^{\prime}\right)+\operatorname{int} R\right)
$$

is convex and we can apply Eidelheit's separation theorem. There exists $\left(y^{*}, z^{*}\right) \in Q^{+} \times$ $R^{+} \backslash\{(0,0)\}$ such that, for all $x^{\prime} \in A$,

$$
\left\langle\left(F\left(x^{\prime}\right)+\operatorname{int} Q\right) \cap V+\operatorname{int} Q, y^{*}\right\rangle+\left\langle G\left(x^{\prime}\right)+\operatorname{int} R, z^{*}\right\rangle \geq\left\langle y, y^{*}\right\rangle .
$$

It is easy to prove, using standard arguments, that for every $v \in \operatorname{int} Q,\left\langle v, y^{*}\right\rangle \geq 0$, hence $\left\langle v, y^{*}\right\rangle \geq 0$ for all $v \in \operatorname{cl}(\operatorname{int} Q) \supset Q$. This proves that $y^{*} \in Q^{+}$. Similarly, $z^{*} \in R^{+}$and, 
moreover,

$$
\left\langle\left(F\left(x^{\prime}\right)+\operatorname{int} Q\right) \cap V, y^{*}\right\rangle+\left\langle G\left(x^{\prime}\right), z^{*}\right\rangle \geq\left\langle y, y^{*}\right\rangle,
$$

for all $x^{\prime} \in A$. Then

$$
\left\langle\operatorname{cl}\left(\left(F\left(x^{\prime}\right)+\operatorname{int} Q\right) \cap V\right), y^{*}\right\rangle+\left\langle G\left(x^{\prime}\right), z^{*}\right\rangle \geq\left\langle y, y^{*}\right\rangle
$$

Since $\operatorname{cl}\left(\left(F\left(x^{\prime}\right)+\operatorname{int} Q\right) \cap V\right)=\operatorname{cl}\left(\left(F\left(x^{\prime}\right)+Q\right) \cap V\right) \supset\left(F\left(x^{\prime}\right)+Q\right) \cap V$, we obtain

$$
\left\langle\left(F\left(x^{\prime}\right)+Q\right) \cap V, y^{*}\right\rangle+\left\langle G\left(x^{\prime}\right), z^{*}\right\rangle \geq\left\langle y, y^{*}\right\rangle
$$

for all $x^{\prime} \in A$. Take $x^{\prime}=x$; since $y \in(F(x)+Q) \cap V$, we have that $\left\langle G(x), z^{*}\right\rangle \geq 0$. But there exists $z \in G(x) \cap(-R)$, hence $\left\langle z, z^{*}\right\rangle \geq 0$ and, on the other hand, $\left\langle z, z^{*}\right\rangle \leq 0$, so $\left\langle z, z^{*}\right\rangle=0$, that is, $0 \in\left\langle G(x), z^{*}\right\rangle$. The proof is complete.

\section{References}

[1] A. Aleman, On some generalizations of convex sets and convex functions, Anal. Numér. Théor. Approx. 14 (1985), no. 1, 1-6.

[2] J.-P. Aubin and H. Frankowska, Set-Valued Analysis, Systems \& Control: Foundations \& Applications, vol. 2, Birkhäuser Boston, Massachusetts, 1990.

[3] H. P. Benson, An improved definition of proper efficiency for vector maximization with respect to cones, J. Math. Anal. Appl. 71 (1979), no. 1, 232-241.

[4] J. M. Borwein, Proper efficient points for maximizations with respect to cones, SIAM J. Control Optimization 15 (1977), no. 1, 57-63.

[5] J. M. Borwein and D. Zhuang, Super efficiency in vector optimization, Trans. Amer. Math. Soc. 338 (1993), no. 1, 105-122.

[6] W. W. Breckner and G. Kassay, A systematization of convexity concepts for sets and functions, J. Convex Anal. 4 (1997), no. 1, 109-127.

[7] H. W. Corley, Optimality conditions for maximizations of set-valued functions, J. Optim. Theory Appl. 58 (1988), no. 1, 1-10.

[8] J. P. Dauer and O. A. Saleh, A characterization of proper minimal points as solutions of sublinear optimization problems, J. Math. Anal. Appl. 178 (1993), no. 1, 227-246.

[9] M. Durea, First and second order optimality conditions for set-valued optimization problems, Rend. Circ. Mat. Palermo 53 (2004), no. 2, 451-468.

[10] , Generalized vector equilibrium problems and applications, Panamer. Math. J. 14 (2004), no. $4,37-49$.

[11] J. B. G. Frenk and G. Kassay, On classes of generalized convex functions, Gordan-Farkas type theorems, and Lagrangian duality, J. Optim. Theory Appl. 102 (1999), no. 2, 315-343.

[12] A. Guerraggio, E. Molho, and A. Zaffaroni, On the notion of proper efficiency in vector optimization, J. Optim. Theory Appl. 82 (1994), no. 1, 1-21.

[13] Y.-W. Huang, Generalized constraint qualifications and optimality conditions for set-valued optimization problems, J. Math. Anal. Appl. 265 (2002), no. 2, 309-321.

[14] T. Illés and G. Kassay, Theorems of the alternative and optimality conditions for convexlike and general convexlike programming, J. Optim. Theory Appl. 101 (1999), no. 2, 243-257.

[15] J. Jahn and A. A. Khan, Existence theorems and characterizations of generalized contingent epiderivatives, J. Nonlinear Convex Anal. 3 (2002), no. 3, 315-330.

[16] Generalized contingent epiderivatives in set-valued optimization: optimality conditions, Numer. Funct. Anal. Optim. 23 (2002), no. 7-8, 807-831. 
[17] J. Jahn and R. Rauh, Contingent epiderivatives and set-valued optimization, Math. Methods Oper. Res. 46 (1997), no. 2, 193-211.

[18] V. Jeyakumar, Convexlike alternative theorems and mathematical programming, Optimization 16 (1985), no. 5, 643-652.

[19] B. Jiménez and V. Novo, A notion of local proper efficiency in the Borwein sense in vector optimisation, ANZIAM J. 45 (2003), no. 1, 75-89.

[20] H. Kuk, T. Tanino, and M. Tanaka, Sensitivity analysis in parametrized convex vector optimization, J. Math. Anal. Appl. 202 (1996), no. 2, 511-522.

[21] Z. F. Li, A theorem of the alternative and its application to the optimization of set-valued maps, J. Optim. Theory Appl. 100 (1999), no. 2, 365-375.

[22] Z. F. Li and G.-Y. Chen, Lagrangian multipliers, saddle points, and duality in vector optimization of set-valued maps, J. Math. Anal. Appl. 215 (1997), no. 2, 297-316.

[23] Z. F. Li and S. Y. Wang, Lagrange multipliers and saddle points in multiobjective programming, J. Optim. Theory Appl. 83 (1994), no. 1, 63-81.

[24] D. T. Luc, Theory of Vector Optimization, Springer-Verlag, Berlin, 1989.

[25] A. Mehra, Super efficiency in vector optimization with nearly convexlike set-valued maps, J. Math. Anal. Appl. 276 (2002), no. 2, 815-832.

[26] J.-P. Penot, Differentiability of relations and differential stability of perturbed optimization problems, SIAM J. Control Optim. 22 (1984), no. 4, 529-551.

[27] W. Song, Lagrangian duality for minimization of nonconvex multifunctions, J. Optim. Theory Appl. 93 (1997), no. 1, 167-182.

[28] A. Taa, Set-valued derivatives of multifunctions and optimality conditions, Numer. Funct. Anal. Optim. 19 (1998), no. 1-2, 121-140.

M. Durea: Faculty of Mathematics, "Alexandru Ioan Cuza" University of Iaşi, Boulevard Carol I, no.11, 700506 Iaşi, Romania

E-mail address: durea@uaic.ro 


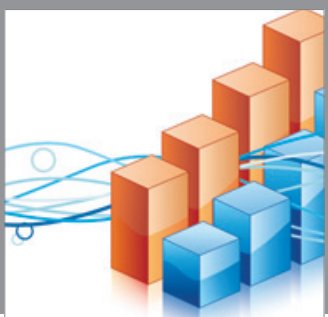

Advances in

Operations Research

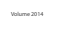

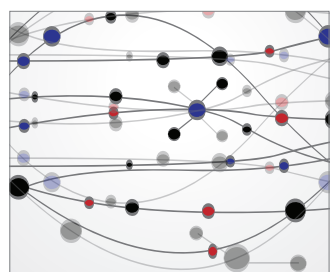

\section{The Scientific} World Journal
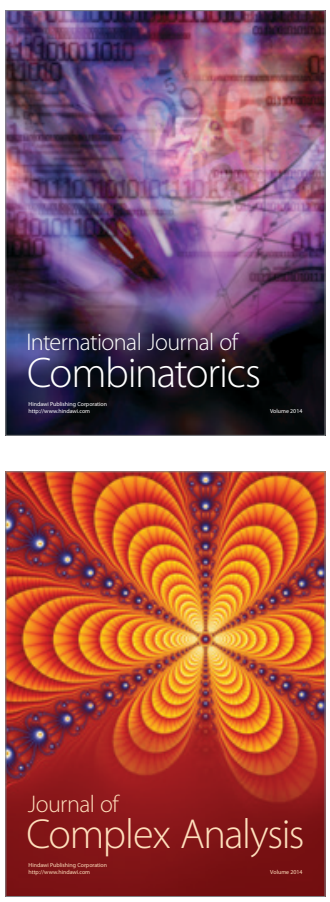

International Journal of

Mathematics and

Mathematical

Sciences
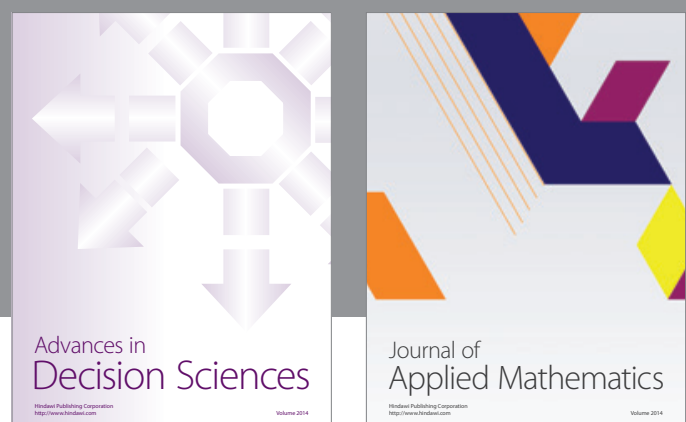

Journal of

Applied Mathematics
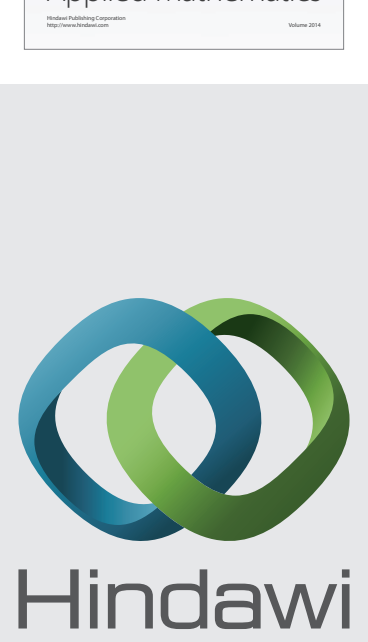

Submit your manuscripts at http://www.hindawi.com
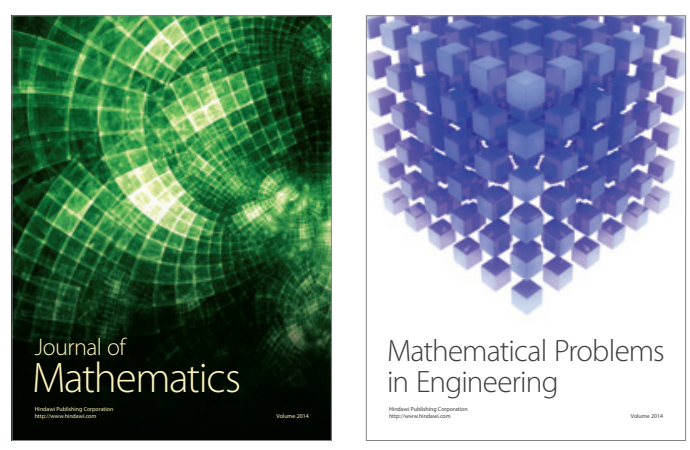

Mathematical Problems in Engineering
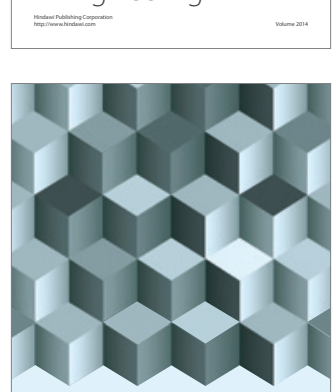

Journal of

Function Spaces
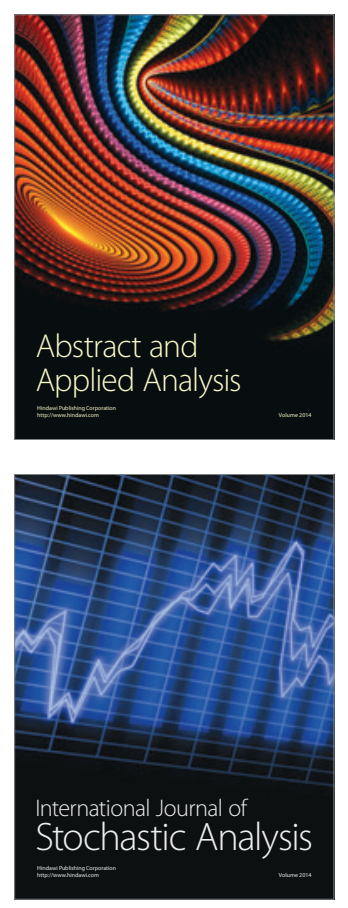

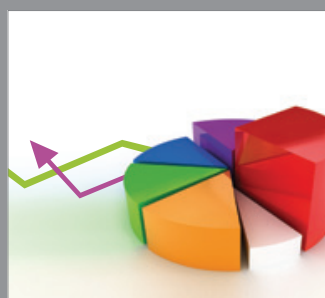

ournal of

Probability and Statistics

Promensencen
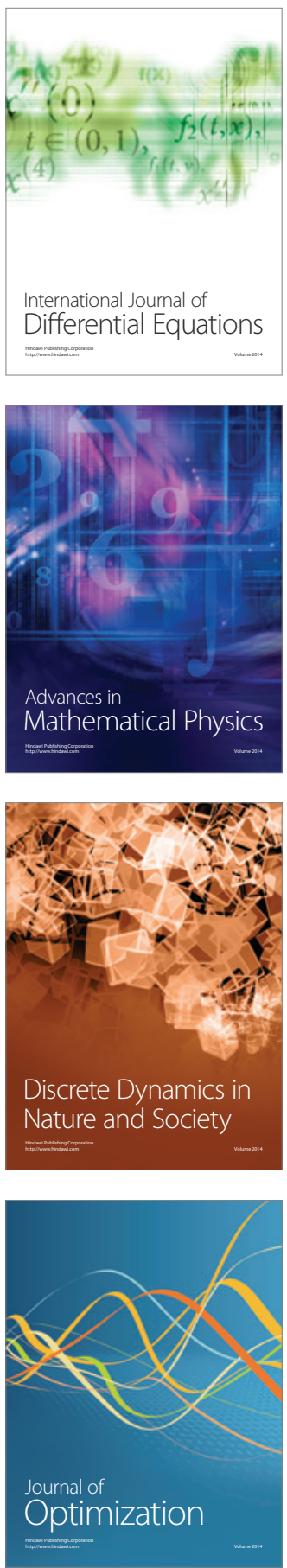